\title{
Presence of Candida cell wall derived polysaccharides in the sera of intensive care unit patients: relation with candidaemia and Candida colonisation
}

\author{
Julien Poissy ${ }^{1,2,3}$, Boualem Sendid ${ }^{1,2,4}$, Sébastien Damiens ${ }^{1,2}$, Ken Ichi Ishibashi ${ }^{5}$, Nadine François ${ }^{4}$, Marie Kauv ${ }^{1,3}$, \\ Raphaël Favory ${ }^{1,3}$, Daniel Mathieu ${ }^{1,3}$ and Daniel Poulain ${ }^{1,2,4^{*}}$
}

\begin{abstract}
Introduction: Prompt diagnosis of candidaemia and invasive candidosis is crucial to the early initiation of antifungal therapy. The poor sensitivity of blood cultures (BCs) has led to the development of fungal glycan tests as a diagnostic adjunct. We analysed the performance of tests for the detection of circulating $\beta$-D-1,3-glucan (BDG) and mannan in the intensive care unit (ICU) setting.

Methods: This retrospective, case-control study included 43 ICU patients with candidaemia and 67 controls, hospitalised on the same ward and assessed weekly for yeast colonisation with simultaneous serum sampling; 340 sera taken before and after positive BCs were available for the cases group and 203 for the controls. BDG and mannan levels were determined using the Fungitell ${ }^{\circledR}$ and Platelia ${ }^{\mathrm{TM}}$ Candida Ag tests, respectively.

Results: BDG was detected early in sera from cases patients but was also present in several sera from controls. Increasing the cut-off from $80 \mathrm{pg} / \mathrm{mL}$ to $350 \mathrm{pg} / \mathrm{mL}$ and $800 \mathrm{pg} / \mathrm{mL}$ resulted in sensitivity/specificity ratios of $0.97 / 0.31,0.65 / 0.74,0.30 / 0.86$, respectively. Detection of mannan was more specific but lacked sensitivity. No obvious correlation was found between BDG and colonisation, but a trend existed between high colonisation and high BDG. Candidaemia relapses were associated with a rise in BDG and mannan but, in contrast to the transient nature of mannan, BDG persisted up to 7 weeks after positive BCs.
\end{abstract}

Conclusion: A combination of mannan and BDG tests could be used to guide pre-emptive therapeutic decisions in ICU patients.

\section{Introduction}

Invasive candidosis (IC) is one of the leading causes of nosocomial infection and Candida species rank fourth among the pathogens involved in bloodstream infections [1]. Despite current progress in research and antifungal therapy, the incidence and attributable mortality of candidaemia remain high [2] due to difficulties in the establishment of an accurate and early diagnosis. In the ICU, candidaemia has a prevalence of 7/1,000 patients, with an attributable mortality of $>40 \%$ compared with $30 \%$ for bacteraemia [3]. Mortality increases from $10 \%$ if antifungal therapy is introduced within 12 hours of the onset of candidaemia to $35 \%$ when treatment is initiated more than 48 hours after [4]. These figures are worse in cases of septic shock due to Candida species [5]. The challenge is therefore to manage the delay in initiation of antifungal treatment, especially as $50 \%$ of cases of IC are not detected by blood cultures (BCs) and 48 hours are generally required for yeast isolation [6]. This low sensitivity of BCs was observed in several large postmortem studies evaluating the sensitivity of $\mathrm{BCs}$ for the diagnosis of deep-seated Candida invasion [7] and was shown to range from $28 \%$ in cases of single organ candidosis to $58 \%$

1Université Lille Nord de France, 1 rue Lefèvre, 59000 Lille, France

${ }^{2}$ INSERM U995-2, Faculté de Médecine-Pôle recherche, Place de Verdun, 59045 Lille, France

Full list of author information is available at the end of the article 
in cases of disseminated IC [8]. Improvement of BC systems has only decreased the delay in yeast isolation for certain species without any improvement in the sensitivity [9]. Relying on BCs or waiting for $\mathrm{BC}$ results is thus not appropriate for managing patients at high risk of IC.

Considering the need for alternatives to BCs for early diagnosis, the Infectious Diseases Society of America and the European Society of Clinical and Microbiology and Infectious Diseases have recommended the use of nonculture-based methods to help make therapeutic decisions $[10,11]$. Among the surrogate markers, some Candida cell-wall-derived polysaccharides or oligosaccharides resulting from their catabolism (glycans) can be detected in the sera of patients with candidosis. These consist of mannan, a polymer of mannose representing the polysaccharide moiety of molecules from the outer cell wall layers, and $\beta$-D-1,3-glucan (BDG), a polymer of glucose making up the fibrils in the middle layers. The combined detection of glycan biomarkers and antimannan antibodies was also recommended in the last Surviving Sepsis Campaign for documentation of the microorganisms involved in septic shock [12]. Numerous studies have evaluated mannan and BDG detection tests for the diagnosis of IC in patients with haematological malignancies and in surgical ICU patients; however, information about the value of glucanaemia and mannanaemia monitoring is scarce.

In this study, we looked at ICU patients with candidaemia and control patients from the same ward and with the same high-risk factors/predisposing conditions for IC with the aim of analysing BDG and mannan levels during hospitalisation in relation to candidaemia onset or Candida colonisation. The primary evaluation measure was an assessment of the two tests to make an early diagnosis of candidaemia. In addition, we analysed how these biomarkers could predict candidaemia relapses or a favourable outcome. Finally, we propose a biomarkerbased algorithm designed especially for the management of ICU patients, most of whom are at high risk of IC.

\section{Materials and methods}

\section{Patients}

This retrospective, case-control study involved adult patients hospitalised in a 50-bed polyvalent ICU department in a tertiary university teaching hospital. The database of the clinical mycology laboratory was screened to select patients with a positive BC for Candida over the period 2005 to 2010 . We focused on patients $>18$ years old for whom sera were available at least 1 week before and 1 week after the day of candidaemia. The control group consisted of patients hospitalised on the same ward with Candida colonisation but no evidence of IC; five body sites (urine, anal swabs, nasal swabs, throat and tracheal aspirates when patients were intubated) were sampled once a week for the semi-quantitative determination of yeast colonisation. The medical files for these patients were analysed retrospectively using a standardised questionnaire to look for arguments for IC based on the criteria previously used by Mohr and colleagues [13] and derived from the European Organisation for Research and Treatment of Cancer/Mycoses Study Group criteria [14]. We also looked for evidence of invasive aspergillosis and infection by Pneumocystis jirovecii and excluded patients who had criteria for these two opportunistic fungal infections.

\section{Blood cultures}

BCs were performed by drawing $10 \mathrm{ml}$ blood from either the peripheral vein or arterial catheters into Mycosis ICF vials incubated at $37^{\circ} \mathrm{C}$ for up to 7 days in a Bactec FX System (Becton Dickinson, Le Pont de Claix, France).

\section{Measurement of $\beta$-D-1,3-glucan in serum}

BDG in serum was measured using the Fungitell ${ }^{\circ}$ kit (Associates of Cape Cod Inc., Falmouth, MA, USA), following the manufacturer's instructions. The recommended cutoff value of $80 \mathrm{pg} / \mathrm{ml}$ was used to define positivity. Samples with BDG levels $>500 \mathrm{pg} / \mathrm{ml}$ were diluted and retested.

\section{Measurement of mannan antigen and anti-mannan antibodies in serum}

Mannan antigen and anti-mannan antibodies were measured using the Platelia ${ }^{\mathrm{mm}}$ Candida Ag (mannan) and Platelia ${ }^{\mathrm{Tm}}$ Candida Ab (mannan Ab) tests (Bio-Rad, Marnes la Coquette, France) according to the manufacturer's instructions. The recommended cutoff values for the mannan and mannan $\mathrm{Ab}$ tests used between 2005 and 2010 were $0.5 \mathrm{ng} / \mathrm{ml}$ and $>10$ AU, respectively. Samples with mannan $>500 \mathrm{pg} / \mathrm{ml}$ were diluted and retested.

\section{Intensity of colonisation}

Colonisation intensity was determined for each date of sampling in the control group. Each sample was incubated on Chromagar (Becton Dickinson, Heidelberg, Germany) medium under standard conditions. Presumptive identification was confirmed by ad hoc tests (Bichrolatex and glabrata RTT, Fumouze Diagnostics, Levallois-Perret, France; API 20C, Biomérieux France, Craponne) and the number of colony-forming units was scored as follows: score $1,<10$ colony-forming units; score 2, 10 to 50 colony-forming units; score 3, >50 colony-forming units; score $4,>50$ colony-forming units confluent. Intensity of colonisation was determined for each date of sampling, by dividing the sum score for each colonised site by the number of sites sampled giving a mean Candida load. An overall score of $>4$ was possible in the case of isolation of several Candida species because we added together the colonisation intensity for each species. 
For the patients with candidaemia, data on colonisation were derived from a systematic weekly survey of urine, nasal, tracheal and anal colonisation for the isolation of multiresistant bacteria, where yeasts were also isolated.

\section{Statistical analysis}

Quantitative variables are expressed as median values and $95 \%$ confidence intervals. Qualitative variables were analysed using Fisher's exact test and quantitative variables using the Wilcoxon test. Tests were two-tailed. Pearson's correlation test was performed when necessary. A significance threshold of 0.05 was retained. All statistical analyses were performed using EpiInfo V3.5.3 (Centers for Disease Control and Prevention). Graphics were drawn using Graphpad Prism6 (GraphPad Software, San Diego, California).

\section{Ethical statement}

All sera used in this study were sampled from patients followed in Lille University Hospital. When no results were available from routine tests, BDG and mannan levels were determined retrospectively from the residual frozen samples. No additional sampling was necessary. As sera were taken from a registered biological collection, patient consent was not required according to French law. Institutional review board approval was given by the Comite de Protection des Personnes Nord-Ouest IV, the ethical committee of our institution.

\section{Results}

\section{Description of the study population}

A total of 117 patients with candidaemia were identified during the study period; 43 (36.8\%) of these had all of the criteria for inclusion in the study. Clinical and demographic data for the candidaemia and control groups are presented in Table 1. The groups did not differ in terms of demography, Simplified Acute Physiology Score and risk factors for Candida infection, except for intestinal surgery, exclusive parenteral nutrition and extrarenal epuration. A significant difference in mortality was recorded during both the ICU stay and the subsequent hospital stay.

The median delay between admission to the ICU and appearance of candidaemia was 19 days (range: 10 to 31 days). Only one Candida species was found in BCs (Candida albicans in 40.5\%, Candida parapsilosis in $23.8 \%$, Candida tropicalis in $19 \%$, Candida glabrata in 16.7\%), except for one patient who had both C. albicans and $C$. parapsilosis. Weekly microbial surveillance of these patients revealed that all were colonised by Candida species. For the control patients, weekly multisite determination of yeast colonisation revealed that all except two patients were colonised with Candida species and approximately one-third was colonised with more than one species: two species in 15 patients, three species in eight patients, and four species in two patients. The relative
Table 1 Major characteristics of patients

\begin{tabular}{|c|c|c|c|}
\hline Characteristic & $\begin{array}{l}\text { Cases } \\
(n=43)\end{array}$ & $\begin{array}{c}\text { Controls } \\
(n=67)\end{array}$ & $\begin{array}{c}P \text { value } \\
\text { (two-tailed) }\end{array}$ \\
\hline Age (years) & $\begin{array}{c}61.0 \\
(50.0 \text { to } 72.5)\end{array}$ & $\begin{array}{c}60.5 \\
(49.5 \text { to } 70.0)\end{array}$ & 0.76 \\
\hline Sex ratio (male/female) & 2.9 & 2.3 & 0.67 \\
\hline Simplify Acute Physiology Score & 51.5 & 53.0 & 0.23 \\
\hline Surgical patient & 37.2 & 20.3 & 0.07 \\
\hline Abdominal surgery & 30.2 & 10.5 & 0.01 \\
\hline Other surgery & 7.0 & 10.5 & 0.7 \\
\hline Deep venous catheter & 97.7 & 96.8 & 1 \\
\hline $\begin{array}{l}\text { Broad-spectrum } \\
\text { antibiotherapy }\end{array}$ & 100 & 98.4 & 1 \\
\hline Corticoids & 30.2 & 39.7 & 0.41 \\
\hline Vasopressor therapy & 65.1 & 57.8 & 0.55 \\
\hline Exclusive parenteral nutrition & 37.2 & 4.7 & $<5 \times 10^{-5}$ \\
\hline Extrarenal epuration & 62.8 & 29.7 & 0.001 \\
\hline $\begin{array}{l}\text { Mechanical invasive } \\
\text { ventilation }\end{array}$ & 100 & 98.4 & 1 \\
\hline $\begin{array}{l}\text { Duration of mechanical } \\
\text { ventilation (days) }\end{array}$ & $\begin{array}{c}31.5 \\
(14.0 \text { to } 47.0)\end{array}$ & $\begin{array}{c}28.5 \\
(15.5 \text { to } 42.0)\end{array}$ & 0.5 \\
\hline Neutropaenia & 7.0 & 6.4 & 1 \\
\hline Antifungal therapy & 86.0 & 18.0 & $<5 \times 10^{-5}$ \\
\hline Fluconazole & 59.5 & 54.5 & \\
\hline Voriconazole & 13.5 & 0 & \\
\hline Caspofungin & 54.1 & 54.6 & \\
\hline Liposomal amphotericin-B & 5.4 & 9.1 & \\
\hline 5-Fluorocytosine & 2.7 & 0 & \\
\hline Bacteraemia & 58.1 & 27.0 & 0.002 \\
\hline \multicolumn{4}{|l|}{ Distribution of bacteria } \\
\hline Gram-positive & 28.0 & 18.8 & \\
\hline Gram-negative & 56.0 & 56.2 & \\
\hline $\begin{array}{l}\text { Mixed Gram-positive and } \\
\text { Gram-negative }\end{array}$ & 16.0 & 25.0 & \\
\hline $\begin{array}{l}\text { ICU duration of } \\
\text { hospitalisation }\end{array}$ & $\begin{array}{c}33.5 \\
(23.5 \text { to } 60.0)\end{array}$ & $\begin{array}{c}29.0 \\
\text { (19.5 to } 46.5)\end{array}$ & 0.5 \\
\hline ICU mortality & 62.8 & 38.5 & 0.02 \\
\hline Hospital mortality & 65.1 & 44.6 & 0.05 \\
\hline
\end{tabular}

Quantitative data are expressed as mean (25th to 75th quartile). Qualitative data are expressed as a percentage.

prevalence of the different Candida species was similar to that in the candidaemia group (in decreasing order: C. albicans, C. parapsilosis, C. glabrata, C. tropicalis).

\section{Glucanaemia and mannanaemia during the ICU stay}

Glucanaemia and mannanaemia in sera from patients with candidaemia are shown in Figure 1A and 1B, respectively, as a function of weeks of hospitalisation before and after positive BCs. Glucanaemia was observed several weeks before positive $\mathrm{BC}$. The median delay between positive BDG and 


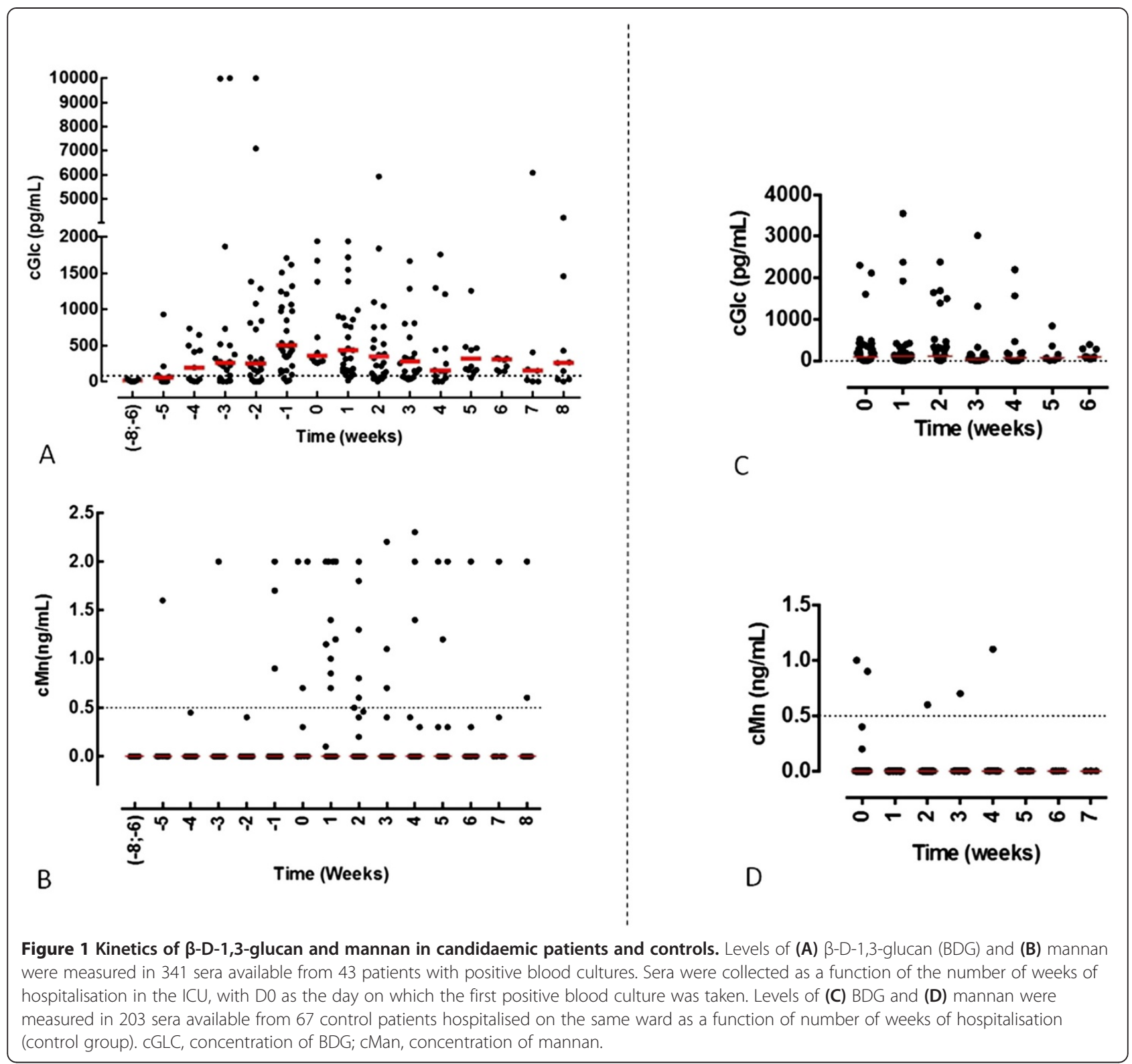

positive $\mathrm{BC}$ was 10 days. The glucan level was maximal the week before positive $\mathrm{BC}$. At the date of positive $\mathrm{BC}$, all sera/ patients had BDG levels $>250 \mathrm{pg} / \mathrm{ml}$. A global decrease in BDG was then observed after week 3, although BDG persisted in some patients for up to 8 weeks.

The data were analysed in terms of sensitivity, specificity and likelihood ratios with reference to the kinetics of BDG. The results are presented in Table 2 for the whole period of serum collection. To respond to the questions 'How can glycanaemia predict the onset of IC?' and 'How can glycanaemia reveal IC?' we considered the period from day 7 before to day 7 after the first positive BC. This analysis was also carried out for patients with negative BCs.
We then analysed how glycanaemia could predict relapse or a favourable outcome by focusing on the 13 patients who had several episodes of candidaemia. Patients were divided into two groups: those with the continuous isolation of yeasts from blood with intervals of $<48$ hours (two BCs for two patients, three BCs for four patients, five $\mathrm{BCs}$ for one patient and nine $\mathrm{BCs}$ for one patient); and patients with well-separated candidaemia episodes corresponding to relapses $(n=5)$. The BDG and mannan $\mathrm{Ab}$ profiles of these patients are shown in Figure 2 and their clinical evolution is described in Additional file 1 . This panel of patients is representative of the diversity of conditions encountered in the ICU and susceptibility to IC. 
Table 2 Sensitivity, specificity and likelihood ratios for glucanaemia and mannanaemia

\begin{tabular}{|c|c|c|c|c|c|c|c|c|}
\hline & \multicolumn{2}{|c|}{ Sensitivity } & \multicolumn{2}{|c|}{ Specificity } & \multicolumn{2}{|c|}{ Positive LR } & \multicolumn{2}{|c|}{ Negative LR } \\
\hline & Week $-8 /+8$ & Day $-7 /+7$ & Week $-8 /+8$ & Day $-7 /+7$ & Week $-8 /+8$ & Day $-7 /+7$ & Week $-8 /+8$ & Day $-7 /+7$ \\
\hline$\beta$-D-1,3-glucan & 100 & 97.1 & 30.6 & 30.6 & 1.4 & 1.4 & 0 & 0.1 \\
\hline Mannan & 38.5 & 32.3 & 95.8 & 95.8 & 9.2 & 7.7 & 0.6 & 0.7 \\
\hline Mannan antibody & 58.1 & 52.9 & 66.2 & 66.2 & 1.7 & 1.6 & 0.6 & 0.7 \\
\hline Mannan or mannan antibody & 79.5 & 58.8 & 66.2 & 64.8 & 2.4 & 1.7 & 0.3 & 0.6 \\
\hline
\end{tabular}

Sensitivity, specificity and positive and negative likelihood ratios (LRs) for glucanaemia ( $\beta$-D-1,3-glucan), mannanaemia and anti-mannan antibody detection tests, as well as a combination of mannan/mannan antibody, during the whole period of serum collection (week 8 before to week 8 after the first positive blood culture (Week $-8 /+8)$ ), and the critical period for diagnosis (day 7 before to day 7 after the first positive blood culture (Day $-7 /+7)$ ).

When analysing the kinetics of BDG, mannan and mannan $\mathrm{Ab}$ in Figure 2, all patients (except Patient 4 with no detectable mannan) had values above the cutoff point for all tests, but with different evolutions. There were positive slopes for BDG and mannan before secondary candidaemia, but different kinetics of circulation in a single patient. The well-known transient circulation of mannan was observed and correlated with the inverse evolution of mannan $\mathrm{Ab}$ (Patients 1, 2, 3 and 5). Interestingly, a sharp decrease in BDG was observed in some patients over a short period of time (see Patients 3, 4 and 5).

Besides the patients described above, the other 25 patients had no documented clinical or mycological signs of relapse. The duration of the survey ranged from day 6 to day 102. At the end of the survey, 21 patients still had BDG above the cutoff value and only four had BDG below the cutoff value. When considering the evolution of the BDG curve, a negative slope was observed for 10 patients, including two who were below the cutoff values at day 9 and day 86, and two who became negative at day 71 and day 74; all other patients still had BDG >150 pg/ $\mathrm{ml}$. Two patients had a positive slope close to $\mathrm{BC}$ isolation (final points at $310 \mathrm{pg} / \mathrm{ml}$ and $760 \mathrm{pg} / \mathrm{ml}$ at day 6 and day 10 , respectively). The other patients $(n=13)$ had stable BDG levels above the cutoff value, including levels as high as 2,223 pg/ml, 1,030 pg/ml and 1,458 pg/ml at day 7, day 12 and day 47, respectively. Median BDG and median duration of the survey were $314 \mathrm{pg} / \mathrm{ml}$ and 18 days, respectively.

\section{Patients hospitalised in the ICU with no evidence of Candida infection}

The results of glucanaemia and mannanaemia tests for the control group are shown in Figure $1 C$ and 1D, respectively, according to the duration of hospitalisation (weeks). As early as the first week of hospitalisation, about $50 \%$ of the patients presented at least one serum sample with BDG above the cutoff value. This percentage remained stable during the following weeks, up to more than 1 month of hospitalisation. By contrast, only five $(2 \%)$ sera samples taken from three different patients had mannanaemia above the cutoff value, and this was transient for all of them.

For the patients followed for Candida colonisation during hospitalisation, glucanaemia was assessed as a function of Candida load at the time of serum sampling. There was no correlation between BDG and yeast load in these patients and 17 (8.4\%) sera samples had values over $1,000 \mathrm{pg} / \mathrm{ml}$ (Additional file 2). As shown in Additional file 3, BDG was significantly associated with a high global Candida load. Analysis of the effect of colonisation on mannan did not show any correlation for the very limited number $(n=3)$ of control patients with mannanaemia.

\section{Impact of variation of the cutoff value on the} performance of $\beta-D-1,3-g l u c a n$ and mannan detection

Receiver operating characteristic curves for BDG and mannan are shown in Figure 3 for the period from day 7 before to day 7 after the first positive BC. For BDG, for a cutoff value of $1,600 \mathrm{pg} / \mathrm{ml}$, sensitivity was 0.05 with a specificity of $>0.9$. For a cutoff value of $800 \mathrm{pg} / \mathrm{ml}$, the respective values were 0.30 and 0.86 . The best sensitivity/ specificity ratio $(0.65 / 0.74)$ was obtained for a cutoff value of $350 \mathrm{pg} / \mathrm{ml}$. For mannan, the best sensitivity/specificity ratio was $0.36 / 0.94$ for a cutoff value of $0.2 \mathrm{ng} / \mathrm{ml}$. From these results it appears that these two tests could be use to complement each other: mannan has an important contribution to specificity for low BDG values, while high BDG levels have improved sensitivity and specificity.

\section{Discussion}

In this study, we evaluated the diagnostic value of glucanaemia in combination with mannanaemia in sera taken sequentially from ICU patients with candidaemia. In parallel, we also assessed the effect of Candida colonisation on serum levels of Candida-derived polysaccharides in patients from the same ICU department who did not have candidaemia. Altogether, 543 sera taken from 110 patients were analysed. The method of inclusion was intended to mimic clinical practice where clinicians have to quickly identify any ICU patients needing antifungal therapy, including those with negative BCs [6].

Glucanaemia was detected several days, or in some cases weeks, before the isolation of Candida species 


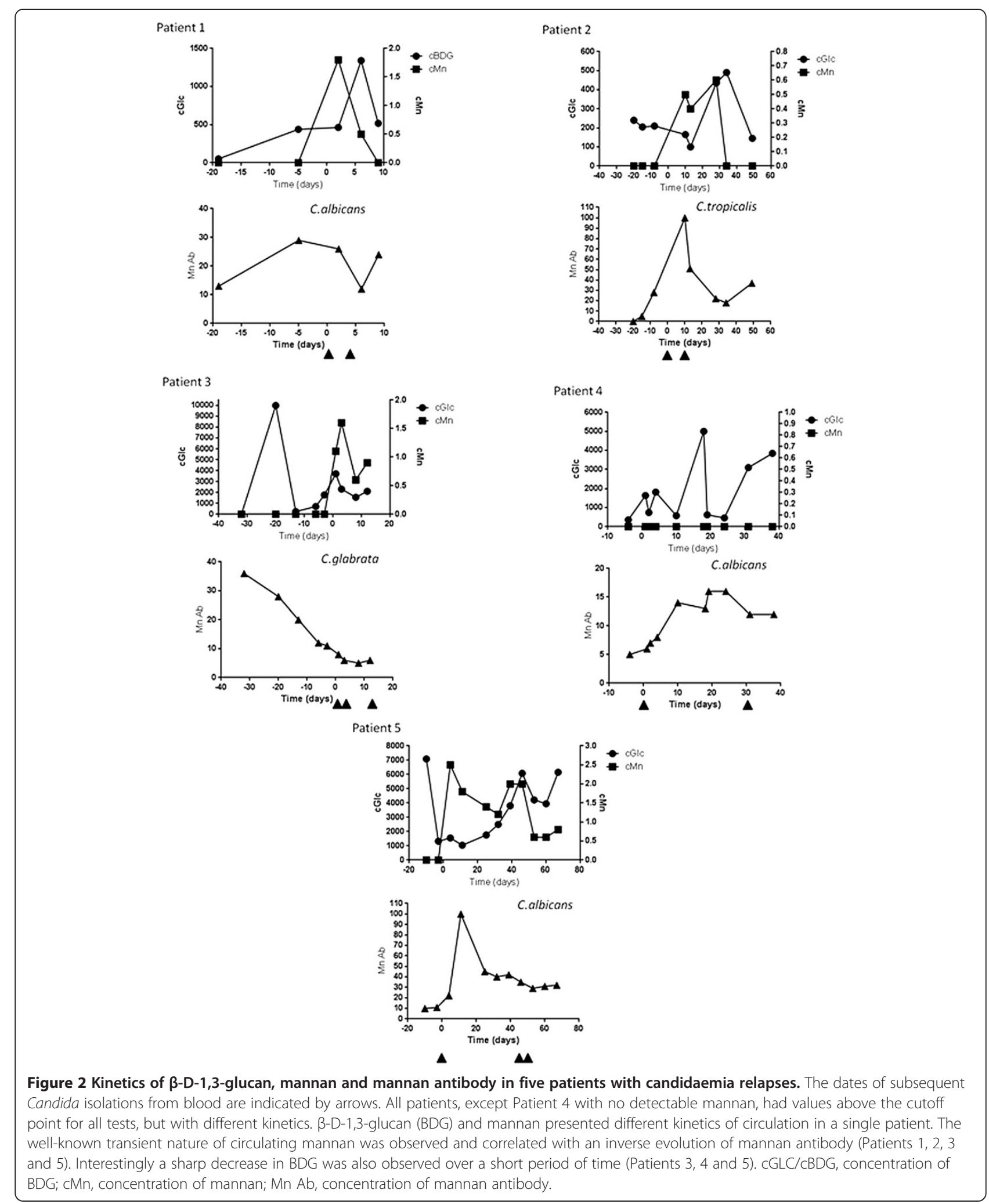

from the blood. In a pilot study evaluating plasma BDG measurement for the diagnosis of deep-seated mycoses and fungal febrile episodes, Obayashi and colleagues reported a sensitivity of $90 \%$ for a cutoff point of $20 \mathrm{pg} /$ $\mathrm{ml}$ [15]. However, subsequent studies gave disparate results from different colorimetric and turbidimetric 


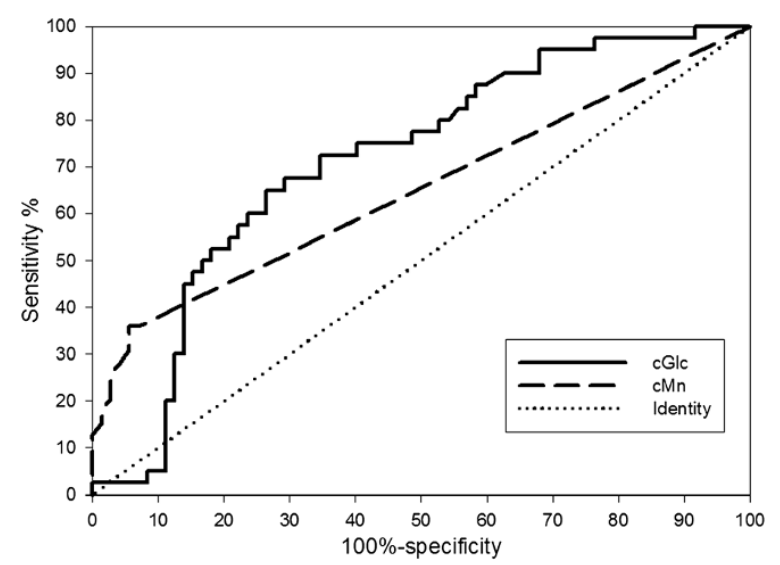

Figure 3 Receiver operating characteristic curves for $\beta$-D-1,3-glucan (plain line) and mannan (dotted line) for the period from day 7 before to day 7 after the first positive blood culture. Area under the curve (95\% confidence interval) for $\beta-D-1$, 3-glucan $(B D G)=0.71$ (0.61 to 0.81) with $P<0.0001$; threshold for the best sensitivity/specificity ratio, 353 (0.65 to 0.74$)$. Area under the curve $(95 \%$ confidence interval) for mannan $=0.63$ ( 0.52 to 0.74 ) with $P=0.009$; threshold for the best sensitivity/specificity ratio, $0.2(0.36$ to 0.94$)$. When considering how the two tests could complement each other, mannan has an important contribution to specificity for low BDG values while higher BDG values have improved sensitivity and specificity. CGLC, concentration of BDG; CMn, concentration of mannan.

methods [16] using glucans as standards, in the absence of knowledge regarding the nature of the molecule(s) detected [17]. With the Fungitell test, the multicentre evaluation by Ostrosky-Zeichner and colleagues, including 107 cases of proven candidosis, reported a sensitivity/specificity ratio of $0.60 / 0.92$ for a cutoff point of $80 \mathrm{pg} / \mathrm{ml}$, although their use of control sera from healthy subjects could have led to an overestimation [18]. Using hospitalised patients from a large hospital study as controls, a similar sensitivity but lower specificity (70\%) was reported [19]; this decreased further in another study involving surgical patients [13].

Impressive sensitivity and specificity (around 0.93 ) for BDG detection for the diagnosis of candidaemia were reported in septic patients with the early stages of fever [20]. This contrasts with the lower performance of this test reported in other studies [19,21], especially low specificity [22]. Indeed, studies published to date report a wide range of sensitivities and specificities for BDG detection [23]. The impact of neutropaenia [24] on BDG circulation is probably worth considering. However, in our cohort, an analysis of sensitivity performed on data collected from patients transferred from a haematology ward suggested that the performance of BDG detection was equivalent to that in non-neutropaenic patients (data not shown). Altogether, the best sensitivity/specificity ratio (0.65/074) for BDG was found for a cutoff value of $350 \mathrm{pg} / \mathrm{ml}$, which is very close to that proposed by Jaijakul and colleagues for predictive efficiency [25].

This study clearly shows that the decrease in BDG over the course of the disease was much slower than the decrease in mannan. The mechanism involved in the catabolism of $\beta$-glucans may involve antibodies participating in their clearance since the existence of human anti$\beta$-glucan antibodies has now been established [26,27], as well as an increase in these antibodies during candidaemia [28]. A second hypothesis for the difference in persistence of BDG and mannan is serum mannosidases that cause natural degradation/turnover of endogenous components; this is not the case for glucans, which, like glucuronoxylomannan of Cryptococcus neoformans, are resistant to degradation and are purely exogenous in nature [29]. From a clinical point of view, the slow decrease in BDG that we observed may limit its use for managing antifungal treatment, a conclusion that contradicts a previous study which proposed the follow-up of glucanaemia kinetics as a tool to evaluate the efficacy of echinocandin therapy [25]. However, some patients with candidaemia enrolled in our study had a second increase from their baseline BDG, associated with high levels of mannan before secondary $\mathrm{BCs}$, suggesting that monitoring of glucanaemia and mannanaemia in patients receiving antifungal therapy is a useful strategy to identify patients with relapses.

The observation of persistently high BDG levels also raises the question of their impact on modulation of the immune response via their interaction with membrane and soluble Pattern Recognition Receptors [30].

In the control group, quantitative snapshot analysis did not reveal a correlation between BDG and yeast burden. However, analysis of cumulative colonisation revealed a trend for an association with BDG $>1,000 \mathrm{pg} / \mathrm{ml}$ (data shown in Additional file 1).

In contrast to BDG, only three control patients had detectable mannan, in agreement with previous conclusions that the detection of mannan with the Platelia test has limited sensitivity but high specificity [11-13]. Here, the unusually high frequency of candidaemia episodes involving C. parapsilosis may also contribute to low sensitivity observed. In contrast to the other more pathogenic Candida species, the mannan epitope detected by the Platelia test is poorly expressed on C. parapsilosis [11]. As in a recent study [31], exclusion of C. parapsilosis resulted in a moderate gain in sensitivity (from 38\% to $45 \%)$. Surprisingly, the specificity of BDG detection was also affected by the exclusion of $C$. parapsilosis cases (from 30\% to 45\%). This is of interest in view of the steady increase in incidence of C. parapsilosis candidaemia [32].

The low sensitivity of mannan detection has been attributed to the circulation of high levels of circulating mannan $\mathrm{Ab}$ and soluble lectins such as mannan-binding lectin forming immune complexes responsible for the 
rapid clearance of mannan [33-35]. This observation has led to the recommendation for repeated serum sampling and combined mannan and mannan Ab screening to improve the overall sensitivity of mannan-based diagnosis [36]. This recommendation has never been reevaluated, but it is interesting to observe here once again the relation of the respective slopes where a sharp decrease in mannan $\mathrm{Ab}$ is often predictive of a mannan peak and vice versa. However, mannan gave the higher positive likelihood ratio during the crucial period from day 7 before to day 7 after the first positive $\mathrm{BC}$ (7.7 vs. 1.4 for BDG).

This study has several limitations, related to its retrospective character and possible selection bias linked to the availability of sera from candidaemia patients and the constitution of the control group. Nevertheless, this latter group was representative of the high colonisation levels encountered in ICU patients, as reported in studies involving surgical ICU patients [37] and medical ICU patients who showed an increase in colonisation as a function of the duration of hospitalisation and cumulative exposure to risk factors [38]. This intense colonisation is itself a risk factor for the development of IC. Indeed, application of Mohr and colleagues' criteria [13] led us to exclude a high proportion of patients from the control cohort, defined as having possible or probable IC. In our opinion, once this selection was made this group represented appropriate controls for ICU daily practice in order to evaluate the performance of biomarkers at discriminating the transition from colonisation to infection, which concerns up to one patient in 10. The differences between the two populations reported in Table 1 probably reflect both the background and the impact of IC $[37,38]$.
From this kinetic analysis, we propose an algorithm (Figure 4) for preemptive treatment based on the principle of biological screening of ICU populations, the majority of whom are at high risk of IC. BDG detection is a more sensitive, early positive test, and therefore appears useful for first-line screening. For BDG values $>800 \mathrm{pg} / \mathrm{ml}$, specificity values $>90 \%$ require no confirmatory test. For a cutoff value $<800 \mathrm{pg} / \mathrm{ml}$ and $>80 \mathrm{pg} / \mathrm{ml}$, determination of mannan is indicated since the high correlation between a combination of these two positive biomarkers and IC could be an indication for preemptive treatment. In other cases, regular monitoring of glucanaemia is indicated since specificity increases by considering two or more successive positive results [13,39] and the cost of serological tests is minimal compared with antifungal treatment administered at excess. The colonisation index should be proposed as a last step despite its usefulness, due to the limitations of time constraints and cost.

The retrospective application of this sequential prescription of BDG and mannan detection in our cohort would have led to the treatment of 24 patients on the basis of BDG values $>800 \mathrm{pg} / \mathrm{ml}$ and of five patients with BDG levels between 80 and $800 \mathrm{pg} / \mathrm{ml}$ associated with positive mannanaemia (total 29 patients $=70 \%$ of patients). When considering early positivity, more than $50 \%$ of the patients $(22 / 41)$ would have been treated before positive BCs. Among the controls, 10 had positive BDG and four had positive mannan $(14 / 67=15 \%)$. These results from the application of our biomarker-based algorithm for preemptive antifungal therapy compare favourably with empiric treatments, which have been shown not to improve the outcome in cases of fluconazole prescription [40] or to be cost-effective [41].

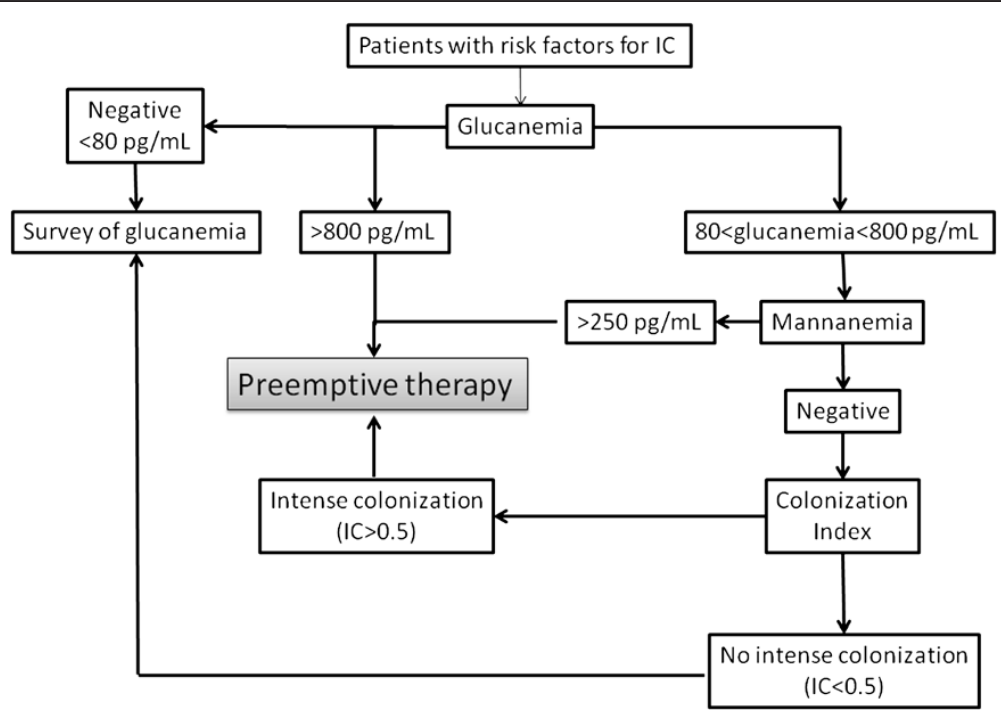

Figure 4 Biomarker-based algorithm proposed for managing preemptive therapy in ICU patients at high risk of developing invasive candidosis. IC, invasive candidosis. 
Similarly, the results compare favourably with probabilist score-based therapy. Indeed, the clinical prediction rule developed by Ostrosky-Zeichner and colleagues captured $10.6 \%$ of the patients with proven/probable IC, but also $34.1 \%$ of patients without IC [42]; the Candida score from Leon and colleagues exhibited an area under the receiver operating characteristic curve of 0.774 , a sensitivity of 77.6 and a specificity of 66.2 [43]. A limitation to this approach is that laboratory procedures often prevent individual testing and, despite the fact that only 2 to 3 hours are needed to obtain the results, sera are often treated as a series, once or twice a week. The periodicity of testing should be adapted for institutions with small numbers of samples or the tests should be performed in a reference centre.

The data presented and discussed here suggest that, due to the lack of sensitivity of BCs, managing therapeutic decisions using the Candida glycan detection test is not unrealistic now that tests are able to detect molecules in the range of picogrammes per millilitre with high reliability. New more sensitive methods for the detection of glycan biomarkers will probably be developed, establishing a panel of tests that cannot be ignored for patient care, especially in the ICU.

\section{Conclusion}

An algorithm for preemptive therapy based on a combination of BDG and mannan detection was derived from a kinetic analysis of a large volume of clinical and biological data collected from patients hospitalised in the same ward and who developed candidaemia or not. The follow-up of BDG and mannan kinetics may also predict relapses. This strategy, which could be adapted to the management of the large number of ICU patients with negative Candida BCs, should be validated through large prospective studies.

\section{Key messages}

- Detection of Candida cell wall polysaccharides in serum is a useful adjunct to $\mathrm{BCs}$ for the diagnosis of IC in the ICU.

- The kinetics and duration of circulation differ between BDG and mannan and between infected patients and colonised controls: BDG is an early sensitive biomarker but has low specificity, while mannan appears later and is less sensitive, but has high specificity.

- Increasing the cutoff value for BDG and combining BDG detection with mannan detection can help in preemptive therapy.

- Mannan and BDG follow-up is not useful for monitoring treatment efficacy, but an increase in these markers is predictive of relapse.

\section{Additional files}

\begin{abstract}
Additional file 1: is a clinical description of patients with relapses of candidaemia, and for whom kinetics profiles of biomarkers are shown in Figure 2.
\end{abstract}

Additional file 2: is a graphical representation of the distribution of glucanaemia reported to the daily fungal load, showing there is no correlation between colonisation and glucanaemia.

Additional file 3: is a graphical representation of the relation between mean global Candida load and glucanaemia, showing a trend for an association between a cumulative high level of colonisation during all of the ICU stay and a high glucanaemia level.

\section{Abbreviations}

Ab: antibody; BC: blood culture; BDG: $\beta-D-1,3-$ glucan; IC: invasive candidosis.

\section{Competing interests}

DP and BS received research grants from Bio-Rad. The remaining authors declare that they have no competing interests.

\section{Authors' contributions}

JP collected data, performed the biological tests, performed the statistical analysis and wrote the paper. BS helped with the selection of sera, helped in the interpretation of the results and wrote the paper. SD participated in the experiments and gave critical analysis in the interpretation of the results. KII participated in the experiments and the analysis of the results. NF helped with the selection of sera from the serum bank and with the biological tests. MK helped to collect clinical data. RF advised on the statistical analysis. DM shared his experience in patient management and helped with the statistical analysis and the development of the algorithm. DP coordinated the work, gave critical advice on the interpretation of the results and wrote the manuscript. All authors read and approved the final manuscript.

\section{Acknowledgements}

The research leading to these results has received funding from the European Community's Seventh Framework Programme (FP7-2007-2013) under HEALTH-F2-2010-260338-ALLFUN and was supported by the Programme Hospitalier de Recherche Clinique du Ministère des Affaires Sociales, de la Santé et de la Ville PHRC 1918, 2011 "Candigène ». The authors thank Val Hopwood for writing assistance.

\section{Author details}

'Université Lille Nord de France, 1 rue Lefèvre, 59000 Lille, France. ${ }^{2}$ INSERM U995-2, Faculté de Médecine-Pôle recherche, Place de Verdun, 59045 Lille, France. ${ }^{3}$ Pôle de Réanimation, CHRU Lille, 2 avenue Oscar Lambret, 59037 Lille Cedex, France. ${ }^{4}$ Laboratoire de Parasitologie Mycologie, Institut de Microbiologie, Pôle de Biologie Pathologie Génétique, rue Paul Nayrac, CHRU Lille, 59037 Lille Cedex, France. ${ }^{5}$ Laboratory for Immunopharmacology of Microbial Products, School of Pharmacy, University of Pharmacy and Life Science, Hachioji, Tokyo 192-0392, Japan.

Received: 14 January 2014 Accepted: 6 June 2014

Published: 29 June 2014

\section{References}

1. Martin GS, Mannino DM, Eaton S, Moss M: The epidemiology of sepsis in the United States from 1979 through 2000. N Engl J Med 2003, 348:1546-1554.

2. Gudlaugsson O, Gillespie S, Lee K, Vande Berg J, Hu J, Messer S, Herwaldt L, Pfaller M, Diekema D: Attributable mortality of nosocomial candidemia, revisited. Clin Infect Dis 2003, 37:1172-1177.

3. Kett DH, Azoulay E, Echeverria PM, Vincent JL: Candida bloodstream infections in intensive care units: analysis of the extended prevalence of infection in intensive care unit study. Crit Care Med 2011, 39:665-670.

4. Morrell M, Fraser VJ, Kollef MH: Delaying the empiric treatment of candida bloodstream infection until positive blood culture results are obtained: a potential risk factor for hospital mortality. Antimicrob Agents Chemother 2005, 49:3640-3645. 
5. Kollef M, Micek S, Hampton N, Doherty JA, Kumar A: Septic shock attributed to Candida infection: importance of empiric therapy and source control. Clin Infect Dis 2012, 54:1739-1746.

6. Clancy CJ, Nguyen MH: Finding the 'missing 50\%' of invasive candidiasis: how nonculture diagnostics will improve understanding of disease spectrum and transform patient care. Clin Infect Dis 2013, 56:1284-1292.

7. Groll AH, Shah PM, Mentzel C, Schneider M, Just-Nuebling G, Huebner K: Trends in the postmortem epidemiology of invasive fungal infections at a university hospital. J Infect 1996, 33:23-32.

8. Berenguer J, Buck M, Witebsky F, Stock F, Pizzo PA, Walsh TJ: Lysis-centrifugation blood cultures in the detection of tissue-proven invasive candidiasis. Disseminated versus single-organ infection. Diag Microbiol Infect Dis 1993, 17:103-109.

9. Meyer MH, Letscher-Bru V, Jaulhac B, Waller J, Candolfi E: Comparison of Mycosis IC/F and plus Aerobic/F media for diagnosis of fungemia by the bactec 9240 system. J Clin Microbiol 2004, 42:773-777.

10. Pappas PG, Kauffman CA, Andes D, Benjamin DK Jr, Calandra TF, Edwards JE Jr, Filler SG, Fisher JF, Kullberg BJ, Ostrosky-Zeichner L, Reboli AC, Rex JH, Walsh TJ, Sobel JD, Infectious Diseases Society of America: Clinical practice guidelines for the management of candidiasis: 2009 update by the Infectious Diseases Society of America. Clin Infect Dis 2009, 48:503-535.

11. Cuenca-Estrella M, Verweij PE, Arendrup MC, Arikan-Akdagli S, Bille J, Donnelly JP, Jensen HE, Lass-Florl C, Richardson MD, Akova M, Bassetti M, Calandra T, Castagnola E, Cornely OA, Garbino J, Groll AH, Herbrecht R, Hope WW, Kullberg BJ, Lortholary O, Meersseman W, Petrikkos G, Roilides E, Viscoli C, Ullmann AJ: ESCMID guideline for the diagnosis and management of Candida diseases 2012: diagnostic procedures. Clin Microbiol Infect 2012, 18:9-18.

12. Dellinger RP, Levy MM, Rhodes A, Annane D, Gerlach H, Opal SM, Sevransky JE, Sprung CL, Douglas IS, Jaeschke R, Osborn TM, Nunnally ME, Townsend SR, Reinhart K, Kleinpell RM, Angus DC, Deutschman CS, Machado FR, Rubenfeld GD, Webb SA, Beale RJ, Vincent JL, Moreno R, Surviving Sepsis Campaign Guidelines Committee including the Pediatric Subgroup: Surviving Sepsis Campaign: international guidelines for management of severe sepsis and septic shock: 2012. Crit Care Med 2013, 41:580-637.

13. Mohr JF, Sims C, Paetznick V, Rodriguez J, Finkelman MA, Rex JH, Ostrosky-Zeichner L: Prospective survey of $(1 \rightarrow 3)$-beta-D-glucan and its relationship to invasive candidiasis in the surgical intensive care unit setting. J Clin Microbiol 2011, 49:58-61.

14. De Pauw B, Walsh TJ, Donnelly JP, Stevens DA, Edwards JE, Calandra T, Pappas PG, Maertens J, Lortholary O, Kauffman CA, Denning DW, Patterson TF, Maschmeyer G, Bille J, Dismukes WE, Herbrecht R, Hope WW, Kibbler CC, Kullberg BJ, Marr KA, Muñoz P, Odds FC, Perfect JR, Restrepo A, Ruhnke M, Segal BH, Sobel JD, Sorrell TC, Viscoli C, Wingard JR, et al: Revised definitions of invasive fungal disease from the European Organization for Research and Treatment of Cancer/Invasive Fungal Infections Cooperative Group and the National Institute of Allergy and Infectious Diseases Mycoses Study Group (EORTC/MSG) Consensus Group. Clin Infect Dis 2008, 46:1813-1821.

15. Obayashi T, Yoshida M, Mori T, Goto H, Yasuoka A, Iwasaki H, Teshima H, Kohno S, Horiuchi A, Ito A, Yamaguchi H, Shimada K, Kawai T: Plasma $(1 \rightarrow 3)$-beta-D-glucan measurement in diagnosis of invasive deep mycosis and fungal febrile episodes. Lancet 1995, 345:17-20.

16. Karageorgopoulos DE, Vouloumanou EK, Ntziora F, Michalopoulos A Rafailidis PI, Falagas ME: $\beta$-D-glucan assay for the diagnosis of invasive fungal infections: a meta-analysis. Clin Infect Dis 2011, 52:750-770.

17. Ueda Y, Ohwada S, Abe Y, Shibata T, lijima M, Yoshimitsu Y, Koshiba T, Nakata M, Ueda T, Kawabata S: Factor G utilizes a carbohydrate-binding cleft that is conserved between horseshoe crab and bacteria for the recognition of beta-1,3-D-glucans. J Immunol 2009, 183:3810-3818.

18. Ostrosky-Zeichner $L$, Alexander BD, Kett DH, Vazquez J, Pappas PG, Saeki F, Ketchum PA, Wingard J, Schiff R, Tamura H, Finkelman MA, Rex JH: Multicenter clinical evaluation of the $(1 \rightarrow 3)$ beta-D-glucan assay as an aid to diagnosis of fungal infections in humans. Clin Infect Dis 2005, 41:654-659.

19. Nguyen MH, Wissel MC, Shields RK, Salomoni MA, Hao B, Press EG, Shields RM, Cheng S, Mitsani D, Vadnerkar A, Silveira FP, Kleiboeker SB, Clancy CJ: Performance of Candida real-time polymerase chain reaction, beta-D-glucan assay, and blood cultures in the diagnosis of invasive candidiasis. Clin Infect Dis 2012, 54:1240-1248.
20. Posteraro B, De Pascale G, Tumbarello M, Torelli R, Pennisi MA, Bello G, Maviglia R, Fadda G, Sanguinetti M, Antonelli M: Early diagnosis of candidemia in intensive care unit patients with sepsis: a prospective comparison of $(1 \rightarrow 3$ )-beta-D-glucan assay, Candida score, and colonization index. Crit Care 2011, 15:R249.

21. Persat F, Ranque S, Derouin F, Michel-Nguyen A, Picot S, Sulahian A: Contribution of the $(1 \rightarrow 3)$-beta-D-glucan assay for diagnosis of invasive fungal infections. J Clin Microbol 2008, 46:1009-1013.

22. Digby J, Kalbfleisch J, Glenn A, Larsen A, Browder W, Williams D: Serum glucan levels are not specific for presence of fungal infections in intensive care unit patients. Clin Diag Lab Immunol 2003, 10:882-885.

23. Theel ES, Doern CD: Point-Counterpoint: $\beta$-D-glucan is an important test in the diagnosis of invasive fungal infections. J Clin Microbiol 2013, 51:3478-3483

24. Senn L, Robinson JO, Schmidt S, Knaup M, Asahi N, Satomura S, Matsuura S, Duvoisin B, Bille J, Calandra T, Marchetti O: 1,3-Beta-D-glucan antigenemia for early diagnosis of invasive fungal infections in neutropenic patients with acute leukemia. Clin Infect Dis 2008, 46:878-885.

25. Jaijakul S, Vazquez JA, Swanson RN, Ostrosky-Zeichner L: (1,3)-Beta-Dglucan as a prognostic marker of treatment response in invasive candidiasis. Clin Infect Dis 2012, 55:521-526.

26. Ishibashi K, Yoshida M, Nakabayashi I, Shinohara H, Miura NN, Adachi Y, Ohno N: Role of anti-beta-glucan antibody in host defense against fungi. FEMS Immunol Med Microbiol 2005, 44:99-109.

27. Chiani P, Bromuro C, Cassone A, Torosantucci A: Anti-beta-glucan antibodies in healthy human subjects. Vaccine 2009, 27:513-519.

28. Sendid B, Dotan N, Nseir S, Savaux C, Vandewalle P, Standaert A, Zerimech F, Guery BP, Dukler A, Colombel JF, Poulain D: Antibodies against glucan, chitin, and Saccharomyces cerevisiae mannan as new biomarkers of Candida albicans infection that complement tests based on C. albicans mannan. Clin Vaccine Immunol 2008, 15:1868-1877.

29. Yauch L, Lam J, Levitz S: Direct inhibition of T-cell responses by the Cryptococcus capsula polysaccharide glucuronoxylomannan. PLOS Pathog 2006, 2:e120.

30. Romani L: Immunity to fungal infections. Nat Rev Immunol 2011, 11:275-288.

31. Held J, Kohlberger I, Rappold E, Busse Grawitz A, Hacker G: Comparison of $(1 \rightarrow 3)$-beta-D-glucan, mannan/anti-mannan antibodies, and Cand-Tec Candida antigen as serum biomarkers for candidemia. J Clin Microbiol 2013, 51:1158-1164.

32. Lortholary O, Desnos-Ollivier M, Sitbon K, Fontanet A, Bretagne S, Dromer F: Recent exposure to caspofungin or fluconazole influences the epidemiology of candidemia: a prospective multicenter study involving 2,441 patients. Antimicrob Agents Chemother 2011, 55:532-538.

33. Sendid B, Tabouret M, Poirot JL, Mathieu D, Fruit J, Poulain D: New enzyme immunoassays for sensitive detection of circulating Candida albicans mannan and antimannan antibodies: useful combined test for diagnosis of systemic candidiasis. J Clin Microbiol 1999, 37:1510-1517.

34. Damiens S, Poissy J, Francois N, Salleron J, Jawhara S, Jouault T, Poulain D, Sendid B: Mannose-binding lectin levels and variation during invasive candidiasis. J Clin Immunol 2012, 32:1317-1323.

35. Sendid B, Poirot JL, Tabouret M, Bonnin A, Caillot D, Camus D, Poulain D: Combined detection of mannanaemia and antimannan antibodies as a strategy for the diagnosis of systemic infection caused by pathogenic Candida species. J Med Microbiol 2002, 51:433-442.

36. Morace $\mathrm{G}$, Borghi E: Fungal infections in ICU patients: epidemiology and the role of diagnostics. Minerva Anestesio/ 2010, 76:950-956.

37. Eggimann P, Bille J, Marchetti O: Diagnosis of invasive candidiasis in the ICU. Ann Intensive Care 2011, 1:37.

38. Charles PE, Dalle F, Aube H, Doise JM, Quenot JP, Aho LS, Chavanet P, Blettery B: Candida spp. colonization significance in critically ill medical patients: a prospective study. Intensive Care Med 2005, 31:393-400.

39. Lamoth F, Cruciani M, Mengoli C, Castagnola E, Lortholary O, Richardson M, Marchetti $O$ : $\beta$-glucan antigenemia assay for the diagnosis of invasive fungal infections in patients with hematological malignancies: a systematic review and meta-analysis of cohort studies from the Third European Conference On Infection in Leukemia (ECIL3). Clin Infect Dis 2012, 54:633-643.

40. Schuster MG, Edwards JE Jr, Sobel JD, Darouiche RO, Karchmer AW, Hadley S, Slotman G, Panzer H, Biswas P, Rex JH: Empirical fluconazole versus placebo for intensive care unit patients: a randomized trial. Ann Intern Med 2008, 149:83-90. 
41. Golan Y, Wolf MP, Pauker SG, Wong JB, Hadley S: Empirical anti-Candida therapy among selected patients in the intensive care unit: a cost-effectiveness analysis. Ann Intern Med 2005, 143:857-869.

42. Ostrosky-Zeichner L, Sable C, Sobel J, Alexander BD, Donowitz G, Kan V, Kauffman CA, Kett D, Larsen RA, Morrison V, Nucci M, Pappas PG, Bradley ME, Major S, Zimmer L, Wallace D, Dismukes WE, Rex JH: Multicenter retrospective development and validation of a clinical prediction rule for nosocomial invasive candidiasis in the intensive care setting. Eur J Clin Microbiol Infect Dis Clin Microbiol 2007, 26:271-276.

43. Leon C, Ruiz-Santana S, Saavedra P, Galvan B, Blanco A, Castro C, Balasini C, Utande-Vazquez A, De Gonzalez De Molina FJ, Blasco-Navalproto MA, López MJ, Charles PE, Martín E, Hernández-Viera MA, Cava Study Group: Usefulness of the 'Candida score' for discriminating between Candida colonization and invasive candidiasis in non-neutropenic critically ill patients: a prospective multicenter study. Crit Care Med 2009, 37:1624-1633

doi:10.1186/cc13953

Cite this article as: Poissy et al:: Presence of Candida cell wall derived polysaccharides in the sera of intensive care unit patients: relation with candidaemia and Candida colonisation. Critical Care 2014 18:R135.

\section{Submit your next manuscript to BioMed Central and take full advantage of:}

- Convenient online submission

- Thorough peer review

- No space constraints or color figure charges

- Immediate publication on acceptance

- Inclusion in PubMed, CAS, Scopus and Google Scholar

- Research which is freely available for redistribution 